\title{
Erratum to: Simulation of the joint effect of rotor-stator interaction and circumferential temperature unevenness on losses in the turbine stage
}

\author{
New title: Modeling the gas-dynamic characteristics of the low-flow and mid-flow \\ model stages for an industrial centrifugal compressor
}

Nicolay Kortikov ${ }^{1, *}$

New authors: Aleksey Borovkov ${ }^{1}$, Igor Voynov $^{1}$, Aleksandr Kirillov ${ }^{1}$, and Aleksandr Drozdov ${ }^{1, *}$

${ }^{1}$ Peter the Great St. Petersburg Polytechnic University, Polytechnicheskaya 29, St. Petersburg, 195251, Russian Federation

Original article: MATEC Web of Conferences 245, 04006 (2018), https://doi.org/10.1051/matecconf/201824504006

The title of the article should be replaced by the following text:

Modeling the gas-dynamic characteristics of the low-flow and mid-flow model stages for an industrial centrifugal compressor

Aleksey Borovkov ${ }^{1}$, Igor Voynov ${ }^{1}$, Aleksandr Kirillov ${ }^{1}$, and Aleksandr Drozdov ${ }^{1, *}$

${ }^{1}$ Peter the Great St. Petersburg Polytechnic University, Polytechnicheskaya 29, St. Petersburg, 195251,

Russian Federation

* Corresponding author: a_drozdi@mail.ru 